\title{
Novas evidências de validade para o Inventário de Estratégias de Coping
}

\author{
New validity evidence for the Coping Strategies Inventory \\ Nuevas evidencias de validez para el Inventario de Estrategias de Afrontamiento
}

\author{
Luana Luca ${ }^{1}$, ORCID 0000-0001-5590-1648 \\ Ana Paula Porto Noronha 2, ORCID 0000-0001-6821-0299 \\ Francine Náthalie Ferraresi Rodrigues Queluz ${ }^{3}$, ORCID 0000-0002-8869-6879 \\ Acácia Aparecida Angeli dos Santos ${ }^{4}$, ORCID 0000-0002-8599-7465 \\ ${ }^{1}$ Centro Universitário de Jaguariúna. Brasil \\ ${ }^{2} 34$ Universidade São Francisco. Brasil
}

\begin{abstract}
Resumo: O objetivo do presente estudo foi analisar as propriedades psicométricas do Inventário de Estratégias de Coping (IEC) por meio de evidências de validade baseadas na estrutura interna e na relação com outras variáveis (forças de caráter). Participaram 927 universitários, com idade média de 26 anos $(D P=7,7)$ e maioria do sexo feminino. Todos responderam ao Inventário de Estratégias de Coping e à Escala de Forças de Caráter (EFC). Após a análise dos dados, sugere-se uma nova organização fatorial com 4 fatores, dos 8 iniciais. Os fatores são: Reavaliação Positiva $(\alpha=0,79)$, Afastamento e Aceitação $(\alpha=0,79)$, Suporte Social $(\alpha=0,67)$ e Confronto e Resolução de Problemas $(\alpha=0,86)$. Os escores do IEC se correlacionaram com os escores da EFC, mostrando que a nova estrutura fatorial encontrada apresenta evidências de validade baseada nas relações com outras variáveis. Os resultados são discutidos à luz da literatura.
\end{abstract}

Palavras-chave: avaliação psicológica; enfrentamento; psicologia positiva; ensino superior; psicometria

Abstract: The aim of the present study was to analyze the psychometric properties of the Coping Strategy Inventory (CCI), through validity evidence based on the internal structure and relationship with other variables (character strengths). Participants were 927 college students, with an average age of 26 years $(S D=7.7)$ and most of them female. All responded to the Coping Strategies Inventory and the Character Strengths Scale (CSE). After analyzing the data, a new factorial organization with four factors, from the initial eight, is suggested. The factors are: Positive Reappraisal $(\alpha=.79)$, Distancing and Acceptance $(\alpha=.79)$, Social Support $(\alpha=.67)$ and Confrontation and Problem Solving $(\alpha=.86)$. The CCI scores correlated with the CSE scores, showing that the new factorial structure found presents validity evidence based on relationships with other variables. The results are discussed in the light of the literature.

Keywords: psychological assessment; coping; positive psychology; higher education; psychometric properties 
Resumen: El objetivo del presente estudio fue analizar las propiedades psicométricas del Inventario de Estrategias de Afrontamiento (IEC) mediante las evidencias de validez basadas en la estructura interna y en la relación con otras variables (fortalezas de carácter). Participaron 927 estudiantes universitarios, con una edad media de 26 años $(D E=7.7)$, la mayoría eran mujeres. Todos respondieron al Inventario de Estrategias de Afrontamiento y la Escala de Fuerzas de Carácter (EFC). Después de analizar los datos, se sugiere una nueva organización factorial de cuatro factores, a partir de los ocho iniciales. Los factores son: Reevaluación positiva $(\alpha=$.79), Retraimiento y aceptación $(\alpha=.79)$, Apoyo social $(\alpha=.67)$ y Confrontación y resolución de problemas $(\alpha=.86)$. Los puntajes del IEC se correlacionaron con los puntajes EFC, mostrando que la nueva estructura factorial encontrada presenta evidencias de validez basadas en relaciones con otras variables. Los resultados se discuten a la luz de la literatura.

Palabras-clave: evaluación psicológica; afrontamiento; psicología positiva; educación superior; psicometría

Recebido: 26/09/2019

Aceito: $15 / 09 / 2020$

Como citar:

Luca, L., Noronha, A.P.P., Queluz, F.N.F.R., \& Angeli dos Santos, A.A. (2020). Novas evidências de validade para o Inventário de Estratégias de Coping. Ciencias Psicológicas, 14(2), e-2319. doi: https://doi.org/10.22235/cp.v14i2.2319

Correspondência: Luana Luca. Centro Universitário de Jaguariúna. Rua Ezio Wagner da Silva, 89, apto 63, Campinas - SP, Brasil. E-mail: luanaluca@gmail.com. Ana Paula Porto Noronha. Universidade São Francisco. Rua Waldemar Cesar da Silveira, 105, Jardim Cura D’ars, Campinas - SP, Brasil. E-mail: ana.noronha8@gmail.com. Francine Náthalie Ferraresi Rodrigues Queluz. E-mail: francine.queluz@gmail.com. Acácia Aparecida Angeli dos Santos; e-mail: acacia.santos@usf.edu.br

\section{Introdução}

Em uma perspectiva cognitivista, Lazarus e Folkman (1984a) definiram coping como um conjunto de esforços emitidos para modificar o ambiente na tentativa de adequar-se da melhor maneira possível a um evento estressor, reduzindo ou minimizando seu caráter aversivo. As mudanças podem ser cognitivas ou comportamentais e servem para que o indivíduo consiga gerenciar situações que excedam seus recursos pessoais. Para os autores, as estratégias de coping podem ser aprendidas e mantidas ou não, no decorrer da vida, dependendo da história de cada um, podendo ser focada no problema ou na emoção.

Para Folkman e Lazarus (1980), o coping focado no problema compreende esforços para identificar o problema, definir soluções e alternativas, avaliar os custos e benefícios das ações, adotar posturas para mudar o que é possível e, se necessário, aprender novas habilidades em relação ao resultado desejado ou esperado. Ao focar no problema, os indivíduos buscam controlar o estressor e as ações são dirigidas para diminuí-lo ou eliminá-lo, sendo consideradas estratégias mais resolutivas.

No que diz respeito às estratégias de coping focadas na emoção, normalmente elas são utilizadas em situações identificadas como imutáveis, sendo mais paliativas. Neste tipo de estratégia, a emoção do indivíduo é modulada diante da situação estressora e, assim, busca-se reduzir a sensação desagradável causada pelo estresse. Por exemplo, quando uma pessoa reza e, com isso, sente-se melhor. No entanto, é importante lembrar que as estratégias de coping estão 
inter-relacionadas, pois pessoas diferentes podem analisar um problema estressor de maneira distinta. Para um indivíduo, a estratégia utilizada para solucionar o problema pode se voltar para a emoção e, para outro, voltar-se ao problema, ou até mesmo, ambas podem ser utilizadas concomitantemente. Um estudante pode estudar para passar em uma prova (problema) e também rezar para conseguir ir bem (emoção) (Folkman, \& Lazarus, 1980).

Ainda, os autores desenvolveram e conceituaram o modelo de avaliação de coping por essas duas formas (problema e emoção) e criaram o Ways of Coping Checklist (WCC), uma medida clínica para medir o coping. O WCC avalia as estratégias de enfrentamento de estresse e as experiências em um contexto clínico, por meio de 67 itens, os quais podem ser respondidos em forma de "sim" ou "não". Posteriormente, Folkman e Lazarus (1988) ampliaram as investigações acerca do instrumento, revisando os dados, que inicialmente eram baseados na literatura, e por meio de uma estratégia empírica propuseram um novo instrumento, o Ways of Coping Questionnaire (WCQ). A principal mudança foi em relação à forma de resposta, que passou de checklist para Likert. O WCQ foi composto por 66 itens da Ways of Coping Checklist, englobando pensamentos, ações e estratégias que as pessoas utilizavam para lidar com demandas internas e externas de um evento estressante específico, com resposta ordinal com quatro possibilidades. Assim os indivíduos deveriam responder indicando a frequência com que usavam cada estratégia de acordo com a seguinte ordenação, 0- não usei; 1 - usei um pouco; 2- usei algumas vezes; 3- usei muitas vezes. A seleção dos itens foi realizada de forma qualitativa, sendo que os que foram considerados pelos seus autores como redundantes ou pouco claros foram excluídos ou revisados e vários itens foram alterados por sugestão dos próprios respondentes (Folkman \& Lazarus, 1988).

Com base no estudo original e em alguns estudos psicométricos do Ways of Coping Questionnaire, foi adotada a subdivisão do instrumento em oito estratégias de coping: Confronto, Afastamento, Autocontrole, Suporte Social, Aceitação de Responsabilidade, Fuga-Esquiva, Resolução de Problemas e Reavaliação Positiva (Coyne, Aldwin, \& Lazarus, 1981; Folkman \& Lazarus, 1985; Folkman, Lazarus, Gruen, \& Delongis, 1986; Folkman \& Lazarus, 1988). A análise da consistência interna dos oito fatores propostos por Lazarus e Folkman (1988), apontou para coeficientes de precisão entre 0,61 (afastamento) e 0,79 (reavaliação positiva).

Outros estudos foram realizados com o intuito de buscar evidências de validade para Ways of Coping Questionnaire. Vitaliano et al. (1985) encontraram uma solução de cinco fatores com 42 itens nos Estados Unidos. Por sua vez, Bramsen et al. (1995), na adaptação do Ways of Coping Questionnaire, para a Holanda, encontraram sete fatores. Na Noruega, Falkum, Oiff e Aasland (1997) identificaram uma estrutura de seis fatores, mantendo os 42 itens de Vitaliano et al. (1985). Mais recentemente, Liew, Santoro, Edwards, Kang e Cronan (2016) adaptaram o instrumento para o contexto de saúde, encontrando uma estrutura de quatro fatores para pacientes estadunidenses com fibromialgia e Corti et al. (2018) encontraram uma estrutura de seis fatores para pacientes australianos com Parkinson. Contudo, o que convém destacar é que as diferenças entre as estruturas fatoriais encontradas são esperadas, uma vez que se trata da adaptação do instrumento para diferentes países e culturas (Borsa \& Seize, 2017).

Apesar das diferentes estruturas fatoriais encontradas, o Ways of Coping Questionnaire é considerado um dos instrumentos mais utilizados para medir estratégias de enfrentamento (Hirsh et al., 2015; Pais-Ribeiro \& Santos, 2001). No Brasil, o instrumento foi traduzido e adaptado por Savóia, Santana e Mejias (1996). Os últimos autores, em seu estudo de tradução e adaptação, nomearam o instrumento como Inventário de Estratégias de Coping (IEC), cuja nomenclatura será adotada neste artigo.

No Brasil, o IEC foi traduzido e adaptado a partir da versão original, com 66 itens e oito fatores, desenvolvida por Folkman e Lazarus (1988). Savóia et al. (1996) verificaram a adequação da tradução do IEC para o português do Brasil, assim como evidências de validade baseadas na estrutura interna e na relação com outras variáveis. Inicialmente foi realizada a tradução do instrumento para o português, que foi submetido à análise de juízes. Estes resultados indicaram 
que a tradução se manteve fiel ao original com relação à interpretação das questões, mesmo considerando as questões culturais e semânticas na tradução.

Com relação à consistência interna do IEC com seus próprios fatores, todas as correlações foram significativas e variaram entre $r=0,42$ e $r=0,68$. Ao utilizar o método teste-reteste, o coeficiente de correlação obtido entre os escores totais, também foi significativo, sendo de $r=0,70$. As evidências de validade em relação a outras variáveis foram investigadas por meio da verificação de correlação entre o IEC e o Inventário de Controle de Estresse de Lipp (1984). Participaram da pesquisa 100 indivíduos que responderam aos dois instrumentos. Os resultados mostraram uma baixa correlação entre os escores totais dos dois instrumentos $(r=0,14, p=0,05)$, no entanto, significativa. Considerando todos esses resultados, os autores concluíram que o instrumento possui qualidades psicométricas satisfatórias (Savóia et al., 1996).

Dinis, Gouveia e Duarte (2011) ampliaram as pesquisas sobre a avaliação de coping e sugeriram uma medida com quatro fatores, a saber: racional, emocional, evitante e distanciado/desligado. Além disso, estudos mais atuais encontraram correlações entre estratégias de coping e características pessoais positivas, tais como com habilidades para resolução de problemas e qualidade de vida de pacientes com esquizofrenia (Park \& Sung, 2016), emoções positivas, resiliência e saúde mental (Gloria \& Steinhardt, 2016), adaptação acadêmica (Luca, Noronha, \& Queluz, 2018) e forças de caráter (Gustems-Carnicer \& Calderón, 2016). As forças de caráter serão utilizadas neste estudo para verificar evidências de validade baseadas na relação com construtos relacionados (American Educational Research Association, American Psychological Association e National Council on Measurement in Education, 2014).

As forças de caráter são compreendidas como características psicológicas positivas, que podem impulsionar o indivíduo, favorecendo o desenvolvimento saudável nos níveis psicológico, biológico e social (Peterson \& Seligman, 2004). Nesse sentindo, a psicologia positiva propõe a existência de 24 forças, sendo elas: amor, amor ao aprendizado, apreciação do belo, autenticidade, autorregulação, bondade, bravura, cidadania, criatividade, curiosidade, esperança, espiritualidade, imparcialidade, inteligência social, gratidão, humor, liderança, modéstia, pensamento crítico, perdão, perseverança, prudência, sensatez e vitalidade (Peterson \& Seligman, 2004).

Harzer e Ruch (2015) buscaram relações entre forças de caráter e coping em um estudo com 214 participantes, sendo 143 do sexo feminino, com idades entre 21 a 64 anos $(M=38,28$ anos; $D P=10,51)$. As forças de caráter intelectuais, emocionais e interpessoais foram positivamente relacionadas com estratégias positivas de enfrentamento. Os autores concluíram que as forças de caráter estão relacionadas a comportamentos de enfrentamento e, assim, funcionam como fator protetivo em relação a situações consideradas estressoras.

Outro ponto que tem sido cada vez mais destacado em estudos mais recentes (Ben-Zur, 2019; Greenaway et al., 2015) refere-se ao aspecto situacional do coping. Neste sentido, é importante que as estratégias de coping sejam avaliadas tendo como referência o contexto em que ocorre o episódio de estresse, das características do próprio evento e das respostas dos indivíduos envolvidos e não determinando as estratégias como sendo boas ou más, adaptativas ou mal adaptativas e isso acontece no IEC. O instrumento mostrou ser uma medida confiável (Savóia et al., 1996), mas suas evidências de validade foram obtidas há mais de 20 anos no Brasil. O Standards for Educational and Psychological Testing (AERA, APA, \& NMCE, 2014) recomenda que as evidências de validade de um instrumento sejam sempre testadas e atualizadas para verificar se as medidas ainda são confiáveis.

Dado o exposto, percebe-se a importância verificar novas evidências de validade para o IEC, além de avaliar se sua estrutura fatorial se mantém. Assim, o objetivo do presente estudo foi analisar as propriedades psicométricas do Inventário de Estratégias de Coping (Folkman \& Lazarus, 1984), adaptado para o português por Savóia et al. (1996), por meio de suas evidências de validade baseadas na estrutura interna (fatorial) e baseadas na relação com outras variáveis relacionadas (forças de caráter), como também avaliar a precisão do instrumento (AERA, APA, \& 
NMCE, 2014). Como hipótese norteadora esperava-se que a estrutura com oito fatores encontrada por Savóia et al. (1996) fosse confirmada e que os escores do Inventário de Estratégias de Coping, tanto geral como nos fatores, se correlacionassem entre si e com as medidas de força de caráter, assim como que o IEC apresentasse boas medidas de precisão.

\section{Método}

\section{Participantes}

Participaram do presente estudo 927 universitários, com idade média de 26 anos $(D P=$ $7,66)$, variando de 18 a 59 anos, sendo $61 \%$ do sexo feminino e maioria solteiro $(69,3 \%)$. Os dados analisados foram de participantes dos cursos de Psicologia (47,9\%), Engenharia $(36,4 \%)$ e Pedagogia (15,8\%). A coleta de dados foi realizada em uma instituição particular do interior de São Paulo (73\%) e outra do estado da Paraíba (27\%). As informações do perfil sociodemográfico dos participantes são apresentados na Tabela 1.

Tabela 1

Perfil Sociodemográfico dos Participantes

\begin{tabular}{llcc}
\hline Variável & & $\boldsymbol{n}$ & $\boldsymbol{\%}$ \\
\hline Sexo & Feminino & 566 & 61,0 \\
& Masculino & 361 & 39,0 \\
\hline \multirow{2}{*}{ Estado Civil } & Solteiro & 633 & 69,3 \\
& Casado/ Relação estável & 261 & 28,6 \\
& Separado/ Divorciado & 19 & 2,1 \\
\hline \multirow{2}{*}{ Curso } & Psicologia & 444 & 47,9 \\
& Engenharia & 337 & 36,4 \\
& Pedagogia & 146 & 15,8 \\
\hline \multirow{2}{*}{ Instituição } & Universidade do estado de São Paulo & 677 & 73,0 \\
& Universidade do estado da Paraíba & 250 & 27,0 \\
\hline
\end{tabular}

\section{Instrumentos}

Inventário de Estratégias de Coping (Folkman \& Lazarus, 1985). O objetivo do IEC é avaliar as estratégias de coping que os indivíduos usam para lidar com as situações adversas do dia-a-dia. No presente estudo, foi utilizada a versão brasileira adaptada por Savóia et al. (1996). O instrumento contém 66 itens que incluem pensamentos e ações utilizados para lidar com demandas internas ou externas de qualquer evento estressor, respondidos por meio de uma escala Likert de quatro pontos. Na versão de Savóia et al. (1996) foram encontrados oito fatores, conforme descrito na introdução, com valores de precisão adequados por meio do teste-reteste (com valores entre $0,40$ e 0,70$)$. Os valores do alfa de Cronbach não foram apresentados pelos autores.

Escala de Forças de Caráter (IFC) (Noronha \& Barbosa, 2016). O objetivo do instrumento é avaliar as 24 forças de caráter, a saber: Amor, Amor ao aprendizado, Apreciação do belo, Autenticidade, Auto-regulação, Bondade, Bravura, Cidadania, Criatividade, Curiosidade, Esperança, Espiritualidade, Gratidão, Humor, Imparcialidade, Inteligência social, Liderança, Modéstia, Pensamento crítico, Perdão, Perseverança, Prudência, Sensatez e Vitalidade. O instrumento é composto por 71 itens, em uma escala Likert, variando de 0 (nada a ver comigo) a 4 (tudo a ver comigo). $O$ instrumento apresenta coeficiente alfa equivalente a 0,93 , indicando alta confiabilidade (Noronha, Dellazzana-Zanon \& Zanon, 2015). 


\section{Procedimentos de coleta de dados}

Primeiro foi solicitada autorização das instituições para a coleta de dados. Em seguida, o projeto foi submetido ao Comitê de Ética da Universidade São Francisco, o qual foi aprovado com Protocolo CAAE 50003715.7.0000.5514. Após a aprovação, os estudantes foram convidados a participar do presente estudo nas universidades nas quais a coleta de dados ocorreu e após o aceite, assinaram o Termo de Consentimento Livre Esclarecido (TCLE). Todos receberam informações acerca dos propósitos do estudo e das questões éticas envolvidas. Os instrumentos foram aplicados em ordem alternada para minimizar o fator aprendizagem e fadiga. As aplicações foram coletivas, em sala de aula, com duração aproximada de 30 minutos.

\section{Procedimento de análise de dados}

Para verificar a distribuição dos escores para cada instrumento, foram calculados a média, o desvio padrão, os valores mínimos e máximos, além de indicadores de curtose e assimetria para cada variável. As análises descritivas foram realizadas pelo Statistical Package for the Social Sciences (SPSS). Todas as variáveis apresentaram uma distribuição normal, segundo a inspeção do número de modas, valores de curtose e assimetria e do teste de normalidade de KolmogorovSmirnov (Marôco, 2014). Para testar o modelo proposto por Savóia et al. (1996) foi realizada uma análise fatorial confirmatória (AFC) no software MPLUS. Os índices de ajuste considerados foram: Comparative Fit Index (CFI $\geq 0,90)$, Root Mean Square Error of Approximation (RMSEA, $\leq$ 0,06; com intervalo de confiança de 90\%), o Tucker-Lewis Index (TLI $\geq 0,95$ ), a significância estatística do teste de Qui-quadrado $(\mathrm{p} \leq 0,05)$ e o Qui-quadrado dividido pelo grau de liberdade (x2/gl < 3) (Hu \&Bentler, 1999). Para a análise fatorial exploratória (AFE), foi utilizado o programa Factor, considerando os seguintes índices de ajuste: Comparative Fit Index (CFI $\geq 0,90)$, Root Mean Square Error of Approximation (RMSEA $\leq 0,06$; com intervalo de confiança de 90\%), Goodness-of-fit index (GFI $\geq 0,90)$ e o Qui-quadrado dividido pelo grau de liberdade $(\mathrm{x} 2 / \mathrm{gl}<3)$ (Hu \& Bentler, 1999).

A precisão do instrumento foi calculada por meio do alfa de Cronbach e para verificar possíveis correlações, foram realizadas análises por meio do teste de correlações de Pearson, uma vez que a amostra apresentou uma distribuição normal. Essas medidas foram calculadas por meio do SPSS. Para o presente estudo, a magnitude das correlações foi classificada em: fraca $(<0,30)$, moderada $(0,30$ a 0,59$)$, forte $(0,60$ a 0,99$)$ ou perfeita $(1,0)$ (Levin \& Fox, 2004).

\section{Resultados}

\section{Evidências de validade baseadas na estrutura interna}

$\mathrm{Na}$ Tabela 2 são apresentados os índices de ajuste obtidos após a realização da análise fatorial confirmatória do modelo do Inventário de Estratégias de Coping proposto por Savóia et al. (1996). De acordo com os resultados observados na Tabela 2, percebe-se que o modelo proposto não apresentou um ajuste adequado ao modelo proposto, uma vez que os valores de referência não estavam dentro dos valores de esperados (Hu \& Bentler, 1999). Desta forma, optou-se por realizar uma análise fatorial exploratória (AFE) com o Inventário de Estratégias de Coping para verificar qual seria a estrutura encontrada em uma amostra brasileira atual.

Tabela 2

Índices de ajuste do modelo do Inventário de Estratégias de Coping proposto por Savóia et al. (1996) encontrados na AFC.

\begin{tabular}{lccccc}
\hline Modelo & $x^{2} / \mathrm{gl}$ & $p$ & CFI & RMSEA & TLI \\
\hline Valor de referência & $<3$ & $\geq 0,05$ & $\geq 0.90$ & $\leq 0.06$ & $\geq 0.95$ \\
\hline Oito Fatores & 4,82 & 0,001 & 0,76 & 0,07 & 0,74 \\
\hline
\end{tabular}


$\mathrm{Na}$ AFE, primeiro observou-se se a matriz de dados era passível da fatoração por meio do valor de Kaiser- Meyer-Olkin (KMO), que foi de 0,90. Como este valor se mostrou adequado (Damásio, 2012), a etapa seguinte foi realizar a retenção dos fatores. O método de extração foi a Robust Diagonally Weighted Least Squares (RDWLS), com método de rotação Promin. A escolha do número do número de fatores foi realizada por meio do resultado da Análise Paralela e do Método de Hull (Ledesma, Ferrando, \& Tosi, 2019). A Análise paralela indicou uma solução com 4 fatores e o Método de Hull indicou uma solução com 2 fatores. A partir disso, foram realizadas AFEs nas duas soluções para verificar qual melhor se encaixava teórica e estatisticamente, considerando os índices de ajuste para cada uma. Em cada AFE, os critérios para exclusão dos itens foram: retirar os itens que não saturassem pelo menos em 0,30 ou que apresentaram saturação similar em mais de um fator, até que nenhum item precisasse ser excluído.

$\mathrm{Na}$ estrutura com dois fatores, na primeira rodada, seis itens foram retirados, pois cinco (3, $5,7,10,36)$ não saturaram em nenhum dos fatores e um (65) apresentou saturação similar em ambos. Na segunda rodada, um item (62) foi retirado por apresentar saturação similar nos dois fatores. Na terceira rodada, nenhum item precisou ser retirado. Ao final, a estrutura com dois fatores apresentou $31,37 \%$ de variância total explicada e todos os índices de ajuste apresentaram valores adequados (Hu \& Bentler, 1999) como pode ser observado na Tabela 3.

$\mathrm{Na}$ estrutura de quatro fatores, na primeira rodada da AFE para este modelo, onze itens (3, $9,29,34,35,48,54,63,64,65$ e 66) foram retirados pois não saturaram o valor mínimo em nenhum fator e quatro itens $(10,26,47,50)$ por terem saturação similar. Na segunda rodada, dois itens foram retirados por apresentarem saturação similar $(51,62)$. Já na terceira rodada, mais dois itens foram retirados, um por não saturar (25) e outro por apresentar saturação similar em 2 fatores (40). Na quarta rodada, o item 43 foi excluído por apresentar saturação similar em dois fatores. Finalmente, na quinta rodada nenhum item precisou ser retirado. Ao final, a estrutura com quatro fatores apresentou $40,64 \%$ de variância total explicada e todos os índices de ajuste apresentaram valores adequados (Hu \& Bentler, 1999).

Ao comparar os dois modelos e considerando os índices de ajuste, a variância total explicada e a coesão teórica proposta por Folkman e Lazarus (1980), optou-se pela solução de quatro fatores. Os fatores foram renomeados a partir da aproximação teórica observada nos agrupamentos. A estrutura final encontrada apresentou 46 itens. $\mathrm{O}$ Fator 1 foi interpretado como espelhando "Reavaliação Positiva" (Itens 55, 56, 57, 58, 59, 60 e 61), o Fator 2 como "Afastamento e aceitação" (itens 4, 5, 6, 11, 12, 13, 14, 16, 17, 24, 32, 33, 37, 41, 44 e 53), o Fator 3 como "Suporte Social" (itens 7, 8, 22, 28, 31, 36, 42 e 45) e o Fator 4 como "Confronto e resolução de problemas" (itens 1, 2, 15, 18, 19, 20, 21, 23, 27, 30, 38, 39, 46, 49 e 52).

Tabela 3

Índices de ajuste obtidos nas AFEs realizadas no presente estudo

\begin{tabular}{lcccc}
\hline Modelo & $x^{2} / \mathrm{gl}$ & CFI & RMSEA & GFI \\
\hline Valor de referência & $<3$ & $\geq 0.90$ & $<0.08$ & $\geq 0.90$ \\
\hline Dois Fatores & 2,22 & 0,97 & 0,03 & 0,99 \\
\hline Quatro fatores & 1,43 & 0,98 & 0,03 & 1,00 \\
\hline
\end{tabular}

Na Tabela 4 são apresentadas as correlações entre o escore total do IEC e seus respectivos fatores. Percebe-se que todas as correlações foram estatisticamente significativas. As correlações de maior magnitude foram as relacionadas aos Escore Total do IEC com todos os seus fatores de forma geral, sendo de magnitude forte. Já o Fator 1 se correlacionou moderadamente com os fatores 2, 3 e 4, assim como o Fator 3 e 4 . As correlações de menor magnitude foram entre o Fator 2 e os fatores 3 e 4 . 
Tabela 4

Correlações entre o Escore Total do Inventário de Estratégias de Coping e os Escores nos Quatro Fatores

\begin{tabular}{llccc} 
& $\begin{array}{c}\text { Fator 1 } \\
\text { Reavaliação } \\
\text { Positiva }\end{array}$ & $\begin{array}{c}\text { Fator 2 } \\
\text { Afastamento e } \\
\text { aceitação }\end{array}$ & $\begin{array}{c}\text { Fator 3 } \\
\text { Suporte Social }\end{array}$ & $\begin{array}{c}\text { Fator 4 } \\
\text { Confronto e resolução de } \\
\text { problemas }\end{array}$ \\
\hline Escore Total IEC & $0,74^{*}$ & $0,69^{*}$ & $0,70^{*}$ & $0,74^{*}$ \\
Fator 1 & - & $0,50^{*}$ & $0,37^{*}$ & $0,33^{*}$ \\
Fator 2 & & - & $0,28^{*}$ & $0,15^{*}$ \\
Fator 3 & & - & $0,51^{*}$ \\
Fator 4 & & & & - \\
${ }^{*} p<0,001$ & & & &
\end{tabular}

No presente estudo, para o Fator 1 ( 7 itens), o $\alpha=0,79$ para o Fator 2 (16 itens), o $\alpha=0,79$, para o Fator 3 (8 itens), o $\alpha=0,67$ e para o Fator 4 (15 itens), o $\alpha=0,86$. O alfa de Cronbach $(\alpha=$ 0,89) geral foi excelente. Todos são considerados valores adequados de precisão (Marôco, 2014).

\section{Evidências de validade baseadas nas relações com outras variáveis}

$\mathrm{Na}$ Tabela 5 são apresentadas as correlações entre os fatores e o escore total do IEC com as forças de caráter. Percebe-se que de maneira geral o Escore Total do IEC apresentou correlações estatisticamente significativas e positivas de magnitude fraca com as forças de caráter, sendo que apenas não apresentou coeficiente significativo com Autorregulação e Perdão. O Fator Reavaliação positiva do IEC apresentou correlações estatisticamente significativas e positiva com Apreciação do belo, Bondade, Bravura, Cidadania, Curiosidade, Espiritualidade, Inteligência social e Sensatez e negativa com Autorregulação, sendo que todas foram de magnitude fraca. Já o Fator Afastamento e aceitação apresentou correlação estatisticamente negativa de magnitude fraca com a maioria das forças de caráter, exceto com Apreciação do belo, Bravura, Criatividade e Sensatez. No Fator Suporte Social todas as forças de caráter também se correlacionaram positivamente com magnitude fraca, com exceção de uma única força de caráter, a Autorregulação. Por fim, no Fator Confronto e Resolução de Problemas, as correlações encontradas foram estatisticamente significativas e positivas com todas as forças de caráter e a maioria dessas correlações foi de magnitude moderada. 
Tabela 5

Correlações entre o Escore Total e Fatores do Inventário de Estratégicas de Coping e o Inventário de Forças de Caráter

\begin{tabular}{|c|c|c|c|c|c|}
\hline $\begin{array}{l}\text { Forças de caráter } \\
\text { (IFC) }\end{array}$ & $\begin{array}{l}\text { Escore } \\
\text { Total IEC }\end{array}$ & $\begin{array}{c}\text { Fator } 1 \text { IEC } \\
\text { Reavaliação } \\
\text { Positiva }\end{array}$ & $\begin{array}{c}\text { Fator } 2 \text { IEC } \\
\text { Afastamento e } \\
\text { aceitação }\end{array}$ & $\begin{array}{l}\text { Fator } 3 \\
\text { IEC } \\
\text { Suporte } \\
\text { Social }\end{array}$ & $\begin{array}{c}\text { Fator } 4 \text { IEC } \\
\text { Confronto e } \\
\text { resolução de } \\
\text { problemas }\end{array}$ \\
\hline Amor & $0,15^{* * *}$ & 0,03 & $-0,14 * * *$ & $0,13 * * *$ & $0,36^{* * *}$ \\
\hline $\begin{array}{l}\text { Amor ao } \\
\text { aprendizado }\end{array}$ & $0,14 * * *$ & $-0,01$ & $-0,19 * * *$ & $0,13^{* * *}$ & $0,43 * * *$ \\
\hline $\begin{array}{l}\text { Apreciação do } \\
\text { belo }\end{array}$ & $0,22 * * *$ & $0,11 * *$ & $-0,05$ & $0,16^{* * *}$ & $0,37 * * *$ \\
\hline Autenticidade & $0,09 * *$ & 0,03 & $-0,09 * *$ & $0,11 * *$ & $0,22 * * *$ \\
\hline Autorregulação & $-0,02$ & $-0,12 * * *$ & $-0,17 * * *$ & $-0,02$ & $0,21 * * *$ \\
\hline Bondade & $0,16^{* * *}$ & $0,07 *$ & $-0,09 * *$ & $0,16^{* * *}$ & $0,31 * * *$ \\
\hline Bravura & $0,18 * * *$ & $0,10 * *$ & $-0,04$ & $0,14 * * *$ & $0,32 * * *$ \\
\hline Cidadania & $0,21 * * *$ & $0,07 *$ & $-0,09 * *$ & $0,21 * * *$ & $0,41 * * *$ \\
\hline Criatividade & $0,18 * * *$ & 0,04 & $-0,06$ & $0,15^{* * *}$ & $0,36^{* * *}$ \\
\hline Curiosidade & $0,18 * * *$ & $0,07 *$ & $-0,09 * *$ & $0,16^{* * *}$ & $0,36^{* * *}$ \\
\hline Esperança & $0,15 * * *$ & 0,02 & $-0,18^{*}$ & $0,10 * *$ & $0,41 * * *$ \\
\hline Espiritualidade & $0,17 * * *$ & $0,15^{* * *}$ & $-0,10^{* *}$ & $0,14 * * *$ & $0,30 * * *$ \\
\hline Gratidão & $0,15 * * *$ & 0,03 & $-0,18 * * *$ & $0,14 * * *$ & $0,37 * * *$ \\
\hline Humor & $0,15 * * *$ & 0,04 & $-0,10 * *$ & $0,18 * * *$ & $0,33 * * *$ \\
\hline Imparcialidade & $0,12 * * *$ & 0,01 & $-0,14 * * *$ & $0,12 * * *$ & $0,32 * * *$ \\
\hline $\begin{array}{l}\text { Inteligência } \\
\text { Social }\end{array}$ & $0,22 * * *$ & $0,10 * *$ & $-0,08^{*}$ & $0,25 * * *$ & $0,35 * * *$ \\
\hline Liderança & $0,14 * * *$ & $-0,02$ & $-0,15 * * *$ & $0,18 * * *$ & $0,38 * * *$ \\
\hline Modéstia & $0,09 * *$ & 0,01 & $-0,15^{* * *}$ & $0,12 * * *$ & $0,26 * * *$ \\
\hline $\begin{array}{l}\text { Pensamento } \\
\text { crítico }\end{array}$ & $0,19 * * *$ & 0,05 & $-0,09 * *$ & $0,16 * * *$ & $0,39 * * *$ \\
\hline Perdão & 0,02 & $-0,04$ & $-0,14 * * *$ & $0,07^{*}$ & $0,19 * * *$ \\
\hline Perseverança & $0,09^{*}$ & $-0,05$ & $-0,22 * * *$ & $0,11 * * *$ & $0,38 * * *$ \\
\hline Prudência & $0,13 * * *$ & 0,04 & $-0,14 * * *$ & $0,13 * * *$ & $0,33 * * *$ \\
\hline Sensatez & $0,20 * * *$ & $0,08 *$ & $-0,04$ & $0,21 * * *$ & $0,31 * * *$ \\
\hline Vitalidade & $0,10 * *$ & $-0,05$ & $-0,20 * * *$ & $0,12 * * *$ & $0,38 * * *$ \\
\hline
\end{tabular}

\section{Discussão}

O objetivo do presente estudo foi analisar as propriedades psicométricas do IEC por meio da verificação de suas evidências de validade baseadas na estrutura interna (fatorial) e baseadas na relação com outras variáveis (forças de caráter), assim como avaliar sua precisão (AERA, APA, \& NMCE). Como hipótese norteadora, esperava-se que a estrutura com oito fatores encontrada por Savóia et al. (1996) fosse confirmada. No entanto, quando o modelo de Savóia et al. (1996) foi testado, os índices de ajuste encontrados não estavam dentro dos valores de referência esperados. Uma hipótese para isto foi o tempo que passou entre a testagem de um modelo em 1996 com a testagem atual, indicando que alguma característica da população pode ter mudado. No que diz respeito à amostra, o estudo de Savóia et al. (1996) foi realizado no estado de São Paulo, enquanto o presente foi realizado em São Paulo e na Paraíba, de modo que as diferenças podem 
ser justificadas em função da inclusão de um novo estado na coleta de dados. Ambos estudos foram realizados com universitários, no entanto, Savóia et al. (1996) fornece apenas o N da amostra, não sendo possível realizar outras comparações ou elencar hipóteses sobre possíveis razões para que o modelo não tenha sido confirmado. Os autores não forneceram dados como idade, gênero, se a instituição era pública ou privada, características socioeconômicas, entre outras possibilidades.

No que diz respeito à estrutura fatorial, uma vez que o modelo de Savóia et al. (1996) não foi confirmado, optou-se por realizar uma nova análise fatorial exploratória para verificar qual estrutura fatorial dos itens seria encontrada. Após rodar a nova análise fatorial exploratória, o modelo que se mostrou mais adequado foi um modelo com quatro fatores: Reavaliação Positiva, Afastamento e Aceitação, Suporte Social e Confronto e Resolução de Problemas. Na nova solução, os índices de ajuste se mostraram adequados, os fatores se correlacionaram significativamente entre si e com o escore geral da IEC e os valores de precisão se mostraram adequados. Todas essas variáveis em conjunto, indicam que a nova estrutura fatorial apresenta evidências de validade baseadas na estrutura interna (AERA, APA, \& NMCE, 2014).

Ao se comparar a estrutura fatorial obtida neste estudo com a estrutura de Savóia et al. (1996) percebe-se que o fator Aceitação de Responsabilidade e o fator Autocontrole foram eliminados na nova análise fatorial exploratória. No fator Autocontrole, o único item que se manteve foi o 14, que foi remanejado para o fator Afastamento e Aceitação na nova versão. O item 14 diz "procurei guardar para mim mesmo os meus sentimentos" se justifica teoricamente no fator que reúne Afastamento e Aceitação. Uma hipótese para os itens do fator Autocontrole terem sido eliminados, em sua quase totalidade, é que podem usar estratégias de enfrentamento de estresse, sem necessariamente ter autocontrole. Também, os itens de Aceitação de Responsabilidade que envolvem o reconhecimento de culpa são requisitos para a manifestação de estratégias de enfrentamento de estresse. Apenas aceitar algumas situações que não podem ser mudadas pode ser uma estratégia em si, mas a responsabilização, principalmente no contexto universitário, exige a realização de outras ações, para além dela, o que pode justificar a extinção do fator (Greenaway et al. 2015).

No que diz respeito aos outros fatores, percebe-se que os de Reavaliação Positiva e Suporte Social se mantiveram e que os fatores Afastamento, Aceitação, Confronto e Resolução de Problemas que eram quatro fatores, se uniram se tornando dois. Os itens do fator Fuga-esquiva se dividiram entre os fatores Reavaliação Positiva e Afastamento e Aceitação na versão atual. Isso é pertinente pois a estratégia de fuga-esquiva pode estar relacionada tanto a um processo de afastamento, quando o indivíduo não quer lidar com algo, como também a um processo de reavaliar a situação para entender se a fuga-esquiva é a melhor opção diante do gerador de estresse (Greenaway et al., 2015).

Após a verificação da nova estrutura fatorial do EIC, foi aferido se ele apresentava evidências de validade baseadas nas relações com outras variáveis (no caso, forças de caráter). Percebe-se, por meio dos resultados, que a maioria das forças de caráter se correlacionou positivamente com o escore total do IEC, mostrando que as estratégias de coping estão relacionadas com a presença das forças de caráter. Estes dados corroboram o estudo de GustemsCarnicer e Calderón (2016) e de Harzner e Ruch (2015), indicando que pessoas com mais forças de caráter têm também mais facilidade em encontrar estratégias de coping para lidar com situações adversas.

No que diz respeito aos fatores, o fator 1 de Reavaliação Positiva se refere à estratégia de enfrentamento dirigida para o controle das emoções que estão relacionadas à tristeza como forma de reinterpretação, crescimento e mudança pessoal a partir da situação conflitante (Lazarus \& Folkman, 1984b). Nota-se que foi o fator com menos correlações significativas, todas de magnitudes fracas. Uma explicação para isto é que como o fator se refere à habilidade de enfrentar emoções relacionadas à tristeza, enquanto as forças de caráter dizem respeito a características de personalidade positivas e mais associadas ao bem-estar subjetivo (Niemiec, 2014). 
O fator Afastamento e Aceitação do IEC apresentou correlação estatisticamente negativa com a maioria das forças de caráter. Os comportamentos relacionados ao fator Afastamento e Aceitação foram negativos, pois se referem a comportamentos de evitação em situações estressoras (Lazarus \& Folkman, 1984b), ao mesmo tempo em que as forças são positivas, e contribuem para que indivíduos e sociedade prosperem (Seider, Jayawickreme, \& Lerner, 2017). Por serem negativos, muitas vezes podem se tornar não adaptativos, indo ao sentido oposto dos preceitos das forças de caráter, uma vez que se as situações adversas fossem enfrentadas, elas poderiam ser contornadas (coping voltado para o problema) (Lazarus \& Folkman, 1984b). Tais índices sugerem que, quanto mais se destacam forças relacionadas às qualidades emocionais melhores são as estratégias de enfrentamento. McCrae e Costa (1986) relataram que as pessoas com altos níveis de bem-estar utilizam comportamentos de coping como a ação racional, a procura de ajuda, o extrair força da adversidade e a fé. Assim as teorias de coping estão baseadas na ideia de que, a fim de lidar com os problemas, as pessoas felizes desencadeiam pensamentos e comportamentos que são adaptáveis e úteis, visto que na média as pessoas infelizes enfrentam as dificuldades de maneira mais destrutivas (Diener, Suh, \& Oishi, 1997; Greenaway, 2015).

O fator Suporte Social do IEC se relacionou positivamente com a maioria das forças de caráter. Esse dado pode ser explicado uma vez que pessoas que apresentam mais forças de caráter conseguem angariar mais suporte social no seu dia a dia ao se tornarem pessoas mais "fáceis" de se conviver. Autorregulação apresentou coeficiente nulo, o que pode ser compreendido pelo fato de que ela envolve a capacidade do indivíduo se comportar de maneira efetiva diante de eventos adversos e ao se pensar no suporte social em si, ele envolve outras pessoas, o que não ocorre necessariamente quando a pessoa faz uso de autorregulação em determinada situação. O exemplo dado anteriormente cabe aqui, um universitário pode decidir acordar mais cedo todos os dias para estudar e ir bem em uma prova (autorregulação de comportamento e também coping focado no problema), mas faz isso individualmente, sem ajuda de outrem, com isso, não teria nenhum suporte social ao se comportar dessa maneira, justificando nesse caso, por exemplo, a falta de correlação com essa força de caráter especificamente.

No que diz respeito ao fator Confronto e Resolução de Problemas, percebe-se que todas as forças de caráter apresentaram correlação estatisticamente significativa e foi o fator com maior número de correlações de magnitude moderada, o que se faz sentido teórico, pois ele retrata o enfrentamento da situação estressante. Assim, a correlação indica que quanto mais desenvolvidas as forças de caráter, mais os indivíduos manejam as situações adversas. Estes dados confirmam os estudos de Harzner e Ruch (2015), e indicam que pessoas que apresentam mais fortemente as forças de caráter tem também mais facilidade em desenvolver estratégias de resolução de problemas e confronto. Enfim, dado o exposto e considerando os resultados obtidos, pode-se postular que o instrumento apresenta também evidências de validade baseada nas relações com outras variáveis (AERA et al., 2014), uma vez que a maioria das forças de caráter se relacionou com as medidas do IEC, confirmando a hipótese inicial deste estudo.

As forças de caráter que não apresentaram correlação foram autorregulação e perdão. A autorregulação emocional é a habilidade de moderar a atenção e os comportamentos oriundos de circunstâncias e eventos distintos (Peterson \& Seligman, 2014), nesse sentido parece que os participantes podem ter estratégias de coping em seu repertório, mas terem dificuldade de autorregular seu comportamento, por exemplo, um estudante pode utilizar estratégias de coping como rezar, cruzar os dedos, fazer pensamento positivo entre outras estratégias para tentar ir bem em uma prova, mas não conseguir se organizar para estudar (autorregulação de comportamento). Em alguma medida, tal dado reafirma a exclusão do fator Autocontrole, por possuírem conteúdos aproximados. Assim, além de seus itens não ficarem retidos no fator de origem (Savóia et al., 1996), a correlação entre autorregulação e o escore total do EIC foi nula.

No caso do perdão, também faz sentido teórico, pois uma pessoa pode ter dificuldade em perdoar as outras, mas as mesmo tempo ter inúmeras estratégias de coping em seu repertório no 
seu dia-a-dia. Ainda, pensando na amostra deste estudo, um universitário que não consegue perdoar seu colega, provavelmente consegue desenvolver estratégias de coping como acordar cedo para estudar, organizar trabalhos em grupo, ter pensamento positivo para se sair bem no contexto acadêmico (Gustems-Carnicer \& Calderón, 2016).

\section{Considerações finais}

Tendo em vista que o objetivo do presente artigo foi investigar as evidências de validade do Inventário de Estratégias de Coping, os resultados encontrados apontam para evidências importantes que possibilitam, por exemplo, que os dados sejam relevantes para compreender a amostra de universitários. Foram encontradas evidências de validade de baseadas na estrutura interna e na relação com outras variáveis, porém a estrutura não corroborou a versão original do instrumento, trazendo uma nova sugestão de estrutura fatorial, uma das maiores contribuições deste estudo. Além disso, este estudo serviu para atualizar o formato do instrumento, pois o último que verificou as propriedades psicométricas foi realizado há mais de vinte anos (Savóia et al., 1996).

No entanto, mesmo diante de resultados positivos, ressalta-se que os dados devem ser considerados com ressalvas, uma vez que a coleta foi realizada somente com estudantes universitários, sendo esta uma limitação. Diante disso, em estudos futuros sugere-se a ampliação da investigação para outros contextos, além do acadêmico e com maior variabilidade de idade também. Sugere-se ainda, que seja verificado se a estrutura fatorial se mantém em outras amostras. Uma outra possibilidade é coletar de outras maneiras, que não sejam apenas auto administrado pelos participantes, podendo ser inclusa a realização de entrevistas para incluir participantes com menor ou nenhuma escolaridade e que não conseguiriam participar sem ajuda.

Por fim, é importante também a análise da relação dos escores do IEC com outras variáveis externas, que possam incluir as medidas de alguns critérios que o teste se propõe a medir, assim como as relações com outros testes que avaliem construtos relacionados já estabelecidos na literatura, como por exemplo, estresse, depressão, ansiedade, entre outras possibilidades. Dado o exposto, conclui-se então que este estudo contribuiu para atualizar as evidências de validade do IEC e que novos estudos são necessários visando a ampliação dessas evidências.

\section{Referências}

American Educational Research Association, American Psychological Association, \& National Council on Measurement in Education (AERA, APA, \& NMCE). (2014). Standards for educational and psychological testing. Washington, EUA: American Educational Research Association.

Ben-Zur H. (2019) Transactional Model of Stress and Coping. In V. Zeigler-Hill \& T. Shackelford (Orgs.), Encyclopedia of Personality and Individual Differences (pp. 1-4). Cham, Springer. doi: 10.1007/978-3-319-28099-8_2128-1

Borsa, J. C., \& Seize, M. M. (2017). Construção e adaptação de instrumentos psicológicos. In B. F. Damásio \& J. C. Borsa (Orgs), Manual de desenvolvimento de instrumentos psicológicos (pp. 21-43). São Paulo, SP: Vetor.

Bramsen, I., Bleiker, E., Eveline, M. A., Triemstra, A. H., Mattanja, Van-Rossum, Sandra M. G. et al. (1995). A Dutch adaptation of the Ways of Coping Questionnaire: Factor stucture and psychometric properties. Anxiety. Stress and Coping an International Journal, 8(4), 337352.

Corti, E. J., Johnson, A. R., Gasson, N., Bucks, R. S., Thomas, M. G., \& Loftus, A. M. (2018). Factor Structure of the Ways of Coping Questionnaire in Parkinson's Disease. Parkinson's Disease, 2018, 1-7. doi:10.1155/2018/7128069. 
Coyne, J. C., Aldwin, C., \& Lazarus, R. S. (1981). Depression and coping in stressful episodes. Journal of Abnormal Psychology, 90, 439-447.

Damásio, B. F. (2012). Uso da análise fatorial exploratória em psicologia. Avaliação Psicológica, 11(2), 213-228.

Dinis, A., Gouveia, J., \& Duarte, C. (2011). Contributos para a Validação da Versão Portuguesa do Questionário de Estilos de Coping. Psychologica, 1(1), 35-62.

Falkum, E., Oiff, M, \& Aasland, O. (1997). Revisiting the factor structure of the Ways of Coping Checklist: a three-dimensional view of the problem-focused coping scale. A study among Norwegian physicians. Personality and Individual Differences, 22(2), 257-267.

Folkman, S., \& Lazarus, R. S. (1980). An analysis of coping in a middle-aged community sample. Journal of health and social behavior, 21(3), 219-239.

Folkman, S., \& Lazarus, R. S. (1985). If it changes it must be a process: A study of emotion and coping during three stages of a college examination. Journal of Personality and Social Psychology, 48, 150-170.

Folkman, S., Lazarus, R. S., Gruen, R., \& DeLongis, A. (1986). Appraisal, coping, health status and psychological symptoms. Journal of Personality and Social Psychology, 50, 571-579.

Folkman, S., \& Lazarus, R. S. (1988). Manual for the ways of coping questionnaire. Palo Alto, CA: Consulting Psychologists Press.

Gloria, C. T., \& Steinhardt, M. A. (2016) Relationships among positive emotions, coping, resilience and mental health. Stress Health, 32(2), 145-156. doi: 10.1002/smi.2589

Greenaway, K. H., Louis, W. R., Parker, S. L., Kalokerinos, E. K., Smith, J. R., \& Terry, D. J. (2015). Measures of Coping for Psychological Well-Being. Measures of Personality and Social Psychological Constructs, 1, 322-351. doi:10.1016/b978-0-12-386915-9.00012-7

Gustems-Carnicer, J., \& Calderón, C. (2016). Virtues and character strengths related to approach coping strategies of college students. Social Psychology of Education, 19(1), 77-95.

Harzer, C., \& Ruch, W. (2015). The relationships of character strengths with coping, work-related stress, and job satisfaction. Frontiers in Psychology, 6, 165. doi: 10.3389/fpsyg.2015.00165.

Hirsch, C. D., Barlem, E. L. D., Almeida, L. K. D., Tomaschewski-Barlem, J. G., Figueira, A. B., \& Lunardi, V. L. (2015). Estratégias de coping de acadêmicos de enfermagem diante do estresse universitário. Revista Brasileira de Enfermagem, 68(5), 783-790.

Hu, L., \& Bentler, P. M. (1999). Cutoff criteria for fit indexes in covariance structure analysis: Conventional criteria versus new99 alternatives. Structural Equation Modeling: A Multidisciplinary Journal, 6(1), 1-55. doi: 10.1080/10705519909540118

Lazarus R. S., \& Folkman S. (1984a). Stress appraisal and coping. New York: Springer.

Lazarus R. S., \& Folkman, S. (1984b). Coping and Adaptation. Em W. D. Gentry (Org.), Handbook of Behavioral Medicine (pp. 282-325). New York: The Guilford Press.

Lazarus, R. S., \& Smith, C. A. (1988). Knowledge and appraisal in the cogmtton-emotion relationship. Cognition and Emotion, 2, 281-300.

Ledesma, R. D., Ferrando, P. J., \& Tosi, J. D. (2019). Uso del Análisis Factorial Exploratorio en RIDEP. Recomendaciones para Autores y Revisores. Revista Iberoamericana de Diagnóstico y Evaluación - e Avaliação Psicológica, 52(3), 173-180. doi: 10.21865/RIDEP52.3.13

Levin, J., \& Fox, J. A. (2004). Estatística para ciências humanas. São Paulo, SP: Pearson.

Liew, C. V., Santoro, M. S., Edwards, L., Kang, J., \& Cronan, T. A. (2016). Assessing the Structure of the Ways of Coping Questionnaire in Fibromyalgia Patients Using Common Factor Analytic Approaches. Pain research \& management, 2016, 7297826. doi:10.1155/2016/7297826

Lipp, M. E. N. (1984). Manual do Inventário de Sintomas de Stress para Adultos de Lipp (ISSL). São Paulo, SP: Casa do Psicólogo. 
Luca, L., Noronha, A. P. P., \& Queluz, F. N. F. R. (2018). Relações entre estratégias de coping e adaptabilidade acadêmica em estudantes universitários. Revista Brasileira de Orientação Profissional, 19(2), 169-176. doi: 1026707/1984-7270/2019v19n2p169

Marôco, J. (2014). Análise estatística com o SPSS statistics. Pêro Pinheiro, Portugal: Report Number.

Noronha, A. P. P., Dellazzana-Zanon, L. L., \& Zanon C. (2015). Internal Structure of the Strengths and Virtues Scale in Brazil. Psico-USF, 20(2), 229-235. doi:10.1590/1413-82712015200204

Noronha, A. P. P., \& Barbosa, A. J. G. (2016). Forças e Virtudes: Escala de Forças de caráter. In C. S. Hutz (Org.), Avaliação em Psicologia Positiva: Técnicas e Medidas (pp. 21-43). São Paulo: CETEPP.

Pais-Ribeiro, J., \& Santos, C. (2001). Estudo conservador de adaptação do Ways of Coping Questionnaire a uma amostra e contexto portugueses. Análise Psicológica, 19(4), 491-502.

Park, S. A., \& Sung, K. M. (2016) Effects on Stress, Problem Solving Ability and Quality of Life of as a Stress Management Program for Hospitalized Schizophrenic Patients: Based on the Stress, Appraisal-Coping Model of Lazarus \& Folkman. Journal of Korean Academy of Nursing, 46(4), 583-597. doi: 10.4040/jkan.2016.46.4.583

Savóia, M. G., Santana, P. R., \& Mejias, N. P. (1996). Adaptação do Inventário de Estratégias de Coping de Folkman e Lazarus para o português. Psicologia USP, 7(1), 183-201.

Seider, S., Jayawickreme, E., \& Lerner, J. V. (2017). Theoretical and Empirical Bases of Character Development in Adolescence: A View of the Issues. Journal of youth and adolescence. doi: 10.1007/s10964-017-0650-3

Vitaliano, P.P., Russo, J., Can, J. E., Maiuro, R.D., \& Becker, J. (1985). Ways of Coping Checklist: Revision and psychometric properties. Multivariate Behavioral Research, 20, 3-26. doi:10.1207/s15327906mbr2001_1.

Participação dos autores: a) Planejamento e concepção do trabalho; b) Coleta de dados; c) Análise e interpretação de dados; d) Redação do manuscrito; e) Revisão crítica do manuscrito.

L.L. contribuiu em a,b,c,d; .A.P.P.N. em a,c,e; F.N.F.R.Q. em c,d,e; A.A.A.S. em c,d.

Editora científica responsável: Dra. Cecilia Cracco 